\title{
Intranasal sufentanil is effective for postoperative analgesia in adults
}

\author{
[L'administration intranasale de sufentanil est efficace pour l'analgésie posto- \\ pératoire chez les adultes]
}

Nathalie Mathieu MD, Nathalie Cnudde MD, Edgard Engelman MD, Luc Barvais MD PhD

Purposes: The aim of this prospective, randomized, doubleblind study was to compare two doses of intranasal sufentanil for postoperative analgesia, titrated according to individual requirements based upon a numeric rating scale (NRS) from 0 to 10 for pain.

Methods: Forty patients, American Society of Anesthesiologists physical status I-II, scheduled for herniorrhaphy or hemorrhoidectomy under general anesthesia, were included when postoperative NRS was $>3$. Nurses used a nasal puff device delivering a constant volume. Patients were randomized into two groups: Group A patients received a dose of $0.025 \mu \mathrm{g} \cdot \mathrm{kg}^{-1} / \mathrm{puff}$, Group B patients a dose of $0.05 \mu \mathrm{g} \cdot \mathrm{kg}^{-1} /$ puff. Puffs were administered as often as needed to obtain NRS $\leq 3$, with an interval time of five minutes. Hemodynamic, respiratory measures and sedation were recorded every five minutes.

Results: The probability of persistence of pain in Group B was consistently lower than in Group A. After 20 min, $20 \%$ of the patients had a NRS score $>3$ in Group B, as opposed to $60 \%$ in Group A. At 60 min, no patient had a NRS > 3 in Group B, whereas there was a probability of $20 \%$ to record a NRS $>3$ for Group A. Hemodynamic, respiratory parameters and sedation remained stable with no intergroup differences.

Conclusions: Nasal administration of $0.050 \mu \mathrm{g} \cdot \mathrm{kg}^{-1} /$ puff sufentanil allowed a NRS $<4$ to be attained within one hour in all patients, with efficacy achieved after $20 \mathrm{~min}$. These findings suggest that the intranasal route is an effective mode of sufentanil administration for immediate postoperative analgesia in adult patients.
Objectif : Notre étude prospective, randomisée et à double insu visait à comparer deux doses intranasales de sufentanil comme analgésie postopératoire, ajustées selon les besoins individuels calculés au moyen d'une échelle d'évaluation numérique (EEN) de la douleur de 0 à 10 .

Méthode: Quarante patients, d'état physique ASA I-II, devant subir une herniorraphie ou une hémorroïdectomie sous anesthésie générale, ont été inclus quand l'EEN était $>3$. Les infirmières ont utilisé un instrument nasal à jet fournissant un volume constant. Les patients ont été répartis en deux groupes : ceux du groupe $A$ ont reçu une dose de $0,025 \mu \mathrm{g}^{\mathrm{kg}} \mathrm{g}^{-1} / \mathrm{jet}$ et ceux du groupe $B, 0,05$ $\mu \mathrm{g}^{\mathrm{kg}} \mathrm{I}^{-1} \mathrm{jet}$. Le médicament a été administré aussi souvent que nécessaire pour obtenir une $E E N \leq 3$, selon un intervalle de cinq minutes. Les mesures hémodynamiques et respiratoires et la sédation ont été notées toutes les cinq minutes.

Résultats: La probabilité d'une douleur persistante chez les patients du groupe $B$ a été constamment plus basse que chez ceux du groupe $A$. Après $20 \mathrm{~min}, 20 \%$ avaient un score d'EEN > 3 dans le groupe $B$, mais $60 \%$ dans le groupe A. À 60 min, aucun patient n'avait une EEN $>3$ dans le groupe $B$, mais il y avait une probabilité de $20 \%$ d'un score d'EEN > 3 dans le groupe $A$. Les paramètres hémodynamiques et respiratoires et la sédation sont demeurés stables sans différence intergroupe.

Conclusion: L'administration nasale de $0,050 \mu \mathrm{g} \cdot \mathrm{kg}^{-1} / \mathrm{jet}$ de sufentanil a permis qu'une $E E N<4$ soit obtenue en moins d'une heure chez tous les patients et l'efficacité atteinte après 20 min. On peut penser que l'administration intranasale de sufentanil est un mode efficace pour l'analgésie postopératoire immédiate chez des adultes.

From the Department of Anesthesiology, Erasme University Hospital, Free University of Brussels, Brussels, Belgium.

Address correspondence to: Dr. Nathalie Mathieu, Department of Anesthesiology, Erasme University Hospital, Route de Lennik 808,

B-1070 Brussels, Belgium. Phone: +32 2555 3919; Fax: +32 2 5554363; E-mail: nmathieu@ulb.ac.be

The investigators do not have any financial relationship with pharmaceutical companies.

Accepted for publication May 17, 2005.

Revision accepted July 28, 2005. 


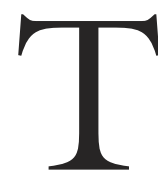

$\mathrm{HE}$ intranasal (IN) route is an attractive, low cost, noninvasive mode for the administration of opioid medications. Transmucosal absorption may offer advantages such as ease of administration, rapid onset, and patient control. Intranasal administration bypasses gastrointestinal and hepatic pre-systemic elimination, and the drug is absorbed directly into the general circulation. ${ }^{1}$

Pharmacokinetic and clinical studies have demonstrated that IN butorphanol ${ }^{2}$ and fentanyl ${ }^{3,4}$ can provide effective pain relief, but only IN fentanyl has been studied for postoperative pain management. Patient-controlled intranasal analgesia with fentanyl is an effective alternative to iv patient-controlled analgesia in postoperative patients. ${ }^{5,6}$

Helmers et al. ${ }^{7}$ have shown that sufentanil, which is two times more lipid soluble than fentanyl, is rapidly and effectively absorbed from the human nasal mucosa, with a bioavailability of $78 \%$. However, most studies have evaluated its use for premedication or sedation using the IN route, and not for postoperative analgesia. ${ }^{7-11}$

Nasal administration of drugs must be done using small volumes to avoid irritation of nasal mucosal membranes. The maximum volume to avoid run-off into the pharynx by a single administration in one nostril in adults is $150 \mu \mathrm{L}$. Individual doses can be adapted by changing the drug concentration, not the volume given, as can be achieved easily when using sufentanil which has a concentration of $50 \mu \mathrm{g} \cdot \mathrm{mL}^{-1}$.

The aim of this prospective, randomized, doublebind study was to evaluate two doses of IN sufentanil for postoperative analgesia in the postanesthesia care unit (PACU), when titrated according to individual requirements based upon an intensity pain scale. The overall goal was to establish the optimal dose of IN sufentanil for the management of moderate pain in the PACU environment.

\section{Methods}

In a preliminary study, we used an IN sufentanil (Janssen-Cilag, Berchem, Belgium) bolus of 0.025 $\mu \mathrm{g} \cdot \mathrm{kg}^{-1}$ and an interval time of $15 \mathrm{~min}$ to control postoperative pain measured by a visual analogue scale (VAS). We concluded that IN sufentanil is a well tolerated postoperative analgesic technique, but the time to obtain adequate analgesia $(V A S<4)$ was more than three hours.

In the present study, we reduced the minimum time between doses to five minutes, taking into consideration the pharmacokinetic profile of IN sufentanil, and the data from our preliminary study.

The study was approved by the local Human Investigation Committee and informed written con- sent was obtained from the patients the day before surgery. We investigated 45 American Society of Anesthesiologists physical status I or II patients aged between 18 and $75 \mathrm{yr}$, scheduled for inguinal hernia repair or hemorrhoidectomy under general anesthesia. Patients with chronic obstructive pulmonary disease, sufentanil allergy, previous nasal surgery, acute or chronic nasal problems, or a history of analgesic abuse were excluded.

A standardized anesthetic was administered consisting of propofol target controlled infusion using diprifusor (Fresinus Vial, Brezins, France), cisatracurium $0.15 \mathrm{mg} \cdot \mathrm{kg}^{-1}$ iv and sufentanil or fentanyl, as preferred by the attending anesthesiologist. The efficacy of two doses of IN sufentanil postoperatively was evaluated, independently of the opioid used during surgery. All patients received propacetamol $2 \mathrm{~g}$ iv and diclofenac $75 \mathrm{mg} i v$ after the induction of anesthesia. This preemptive analgesia regimen is the usual postoperative pain management technique in our institution.

The patients were investigated during the PACU stay until discharge.

Postoperative pain intensity was evaluated using an 11 -point numerical rating scale (NRS; $0=$ no pain and 10 = worst pain possible). In the PACU, the patients were included when the postoperative NRS score was $>3$.

Forty-five patients were investigated. The patients were randomly assigned to one of two groups: Group A: the IN dose $0.025 \mu \mathrm{g} \cdot \mathrm{kg}^{-1}$ and Group B: the IN dose $0.050 \mu \mathrm{g} \cdot \mathrm{kg}^{-1}$.

The volume delivered by the delivery device (Gaasch Packaging, Mollem, Belgium) was constant (0.109 \pm $0.003 \mathrm{~mL}$ ), and the stability of delivering volume was assured in all positions of the receptacle during 48 hr at ambient temperature. The accuracy of the puff volumes were studied by our pharmacy. Four delivery devices (numbered 1, 2, 3, 4) with respectively 30, 20, 10 and $5 \mathrm{~mL}$ were prepared. These devices were stocked at ambient temperature, vertically or horizontally. The resulting volumes of each puff, frequently measured between zero and $48 \mathrm{hr}$ are given in Table I. These results are presented with their mean and standard deviation.

The solution for the delivery device (Figure 1) was prepared by an anesthesiologist who was independent to this protocol. The preparation consisted of the receptacle volume $=10 \mathrm{~mL}$; sufentanil $=50 \mu \mathrm{g} \cdot \mathrm{ml}^{-1}$ :

\section{Group A:}

[Weight of patient $\times 5$ ] $/ 100=\mathrm{X} \mathrm{mL}$ of sufentanil added to the receptacle with $(10-X) \mathrm{mL}$ of sterile water 
TABLE I Volume delivered by the device

\begin{tabular}{lllllll}
\hline Time $(\mathrm{hr})$ & Spray l $(\mathrm{mL})$ & Spray 2 $(\mathrm{mL})$ & Spray 3 $(\mathrm{mL})$ & Spray 4 $(\mathrm{mL})$ & Mean & Standard deviation \\
\hline 0.25 & 0.118 & 0.104 & 0.111 & 0.107 & 0.110 & 0.006 \\
0.50 & 0.118 & 0.117 & 0.096 & 0.112 & 0.111 & 0.010 \\
1 & 0.119 & 0.119 & 0.088 & 0.096 & 0.106 & 0.016 \\
1 & 0.115 & 0.100 & 0.113 & 0.110 & 0.110 & 0.007 \\
2 & 0.121 & 0.117 & 0.116 & 0.106 & 0.115 & 0.006 \\
21 & 0.108 & 0.104 & 0.109 & 0.109 & 0.106 & 0.003 \\
24 & 0.114 & 0.110 & 0.101 & 0.109 & 0.107 & 0.008 \\
25 & 0.110 & 0.105 & 0.110 & 0.113 & 0.109 & 0.005 \\
44 & 0.104 & & 0.108 & 0.004 \\
\hline
\end{tabular}

Four delivery devices (numbered $1,2,3,4$ ) with respectively $30,20,10$ and $5 \mathrm{~mL}$ were prepared. These devices were stocked at ambient temperature, vertically or horizontally. The resulting volumes of each puff, frequently measured between 0 and $48 \mathrm{hr}$ are given. These results are presented as mean and standard deviation. The volume delivered was constant $(0.109 \pm 0.003 \mathrm{~mL})$, and the stability of delivering volume is assured in all positions of the receptacle during $48 \mathrm{hr}$.

TABLE II Patient characteristics

\begin{tabular}{|c|c|c|c|}
\hline & Group $A$ & Group B & P value \\
\hline \multicolumn{4}{|l|}{ Gender* } \\
\hline female & 9 & 13 & \\
\hline male & 11 & 17 & 0.082 \\
\hline Age $($ mean $\pm S D)$ & $51 \pm 13.3$ & $45.8 \pm 18.6$ & 0.16 \\
\hline Weight $($ mean $\pm S D)$ & $72 \pm 12.5$ & $71.5 \pm 11.2$ & 0.64 \\
\hline \multicolumn{4}{|l|}{ Surgery* } \\
\hline inguinal hernia & 5 & 7 & 0.73 \\
\hline hemorroidectomy & 15 & 13 & \\
\hline \multicolumn{4}{|l|}{ Duration of surgery (min) } \\
\hline$($ mean $\pm S D)$ & $87.5 \pm 45.1$ & $82 \pm 38.3$ & 0.72 \\
\hline \multicolumn{4}{|l|}{ Peroperative opioid } \\
\hline Fentanyl* & 3 & 5 & 0.88 \\
\hline Quantity $(\mu \mathrm{g} ;$ mean $\pm \mathrm{SD})$ & $200 \pm 50$ & $260 \pm 147.5$ & 0.53 \\
\hline Sufentanil* & 17 & 15 & 0.19 \\
\hline Quantity $(\mu \mathrm{g} ;$ mean \pm SD $)$ & $31.2 \pm 17.9$ & $24.3 \pm 16$ & 0.273 \\
\hline \multicolumn{4}{|l|}{ NRS Pain $($ mean $\pm S D)$} \\
\hline At arrival PACU & $2.8 \pm 3.5$ & $3.7 \pm 2.5$ & 0.4 \\
\hline At beginning protocol & $5.5 \pm 1.7$ & $5.3 \pm 1.2$ & 0.7 \\
\hline
\end{tabular}

\section{Group B:}

[Weight of patient $\times 10] / 100=\mathrm{X} \mathrm{mL}$ of sufentanil added to the receptacle with $(10-\mathrm{X}) \mathrm{mL}$ of sterile water

A PACU nurse, blinded to the drug, used the delivering device for IN sufentanil administration according to the patient's request. After asking the patient to rate his/her pain intensity on the NRS score, we administered one puff every five minutes to obtain a NRS $\leq 3$ within the first postoperative hour. No maximal dose was provided in the protocol. If the NRS remained $>3$ after the first hour, a rescue therapy using morphine 2 $\mathrm{mg} i v$ boluses with a lockout time of five minutes was given to obtain a pain NRS score $\leq 3$.
Systolic blood pressure, diastolic blood pressure, heart rate, pulse oximetry, and respiratory rate were recorded every five minutes. Evaluation of pain intensity (and analgesic effect), using a NRS, was also recorded every five minutes. Sedation score using the Ramsay sedation scale was recorded every five minutes: $(1=$ anxious and agitated; $2=$ cooperative, tranquil, oriented; 3 = responds only to verbal commands; $4=$ asleep with brisk response to light stimulation; $5=$ asleep without response to light stimulation; $6=$ non responsive). The intensity of nausea was evaluated on an 11 -point nausea rating scale, where: $0=$ no nausea and $10=$ unbearable nausea. Evaluation of the sedation level by the Ramsay sedation scale, the comfort 


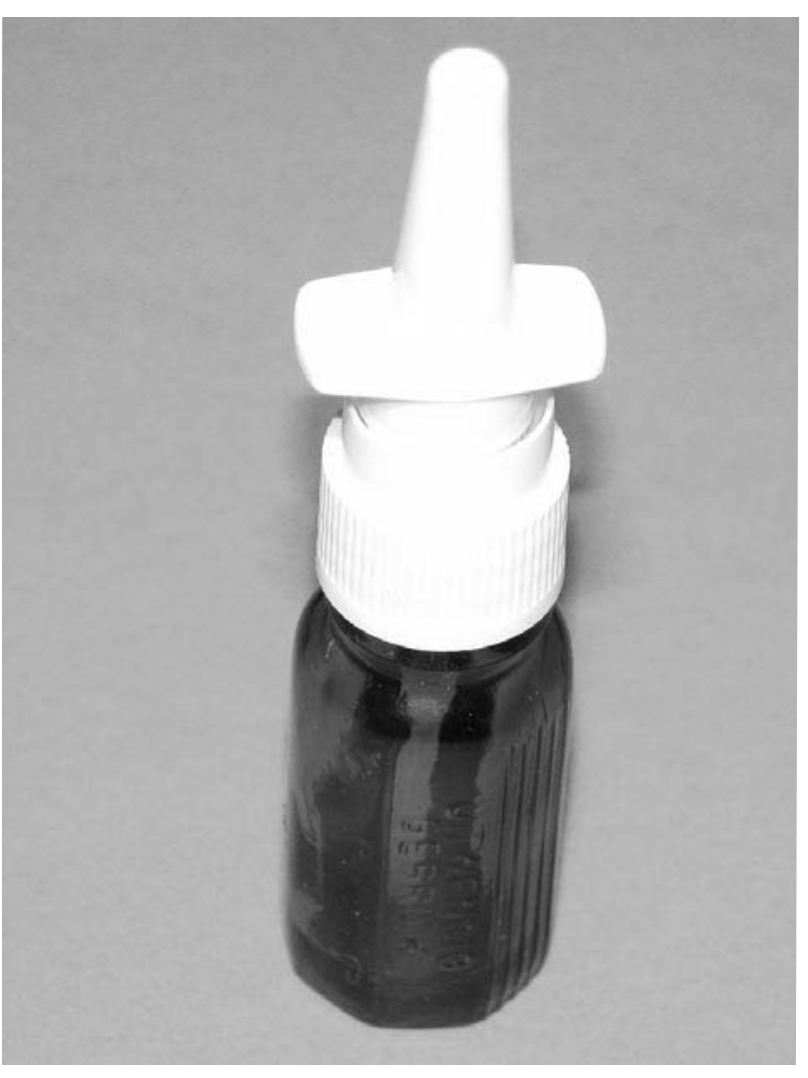

FIGURE 1 Delivery device used for the study.

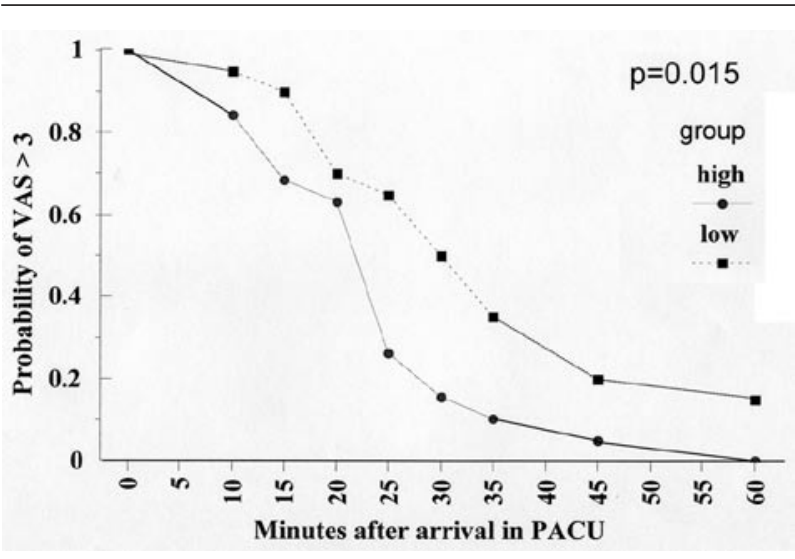

FIGURE 2 The probability of persistence of numerical rating scale $>3$ is shown: graphic using the Kaplan-Meier method.

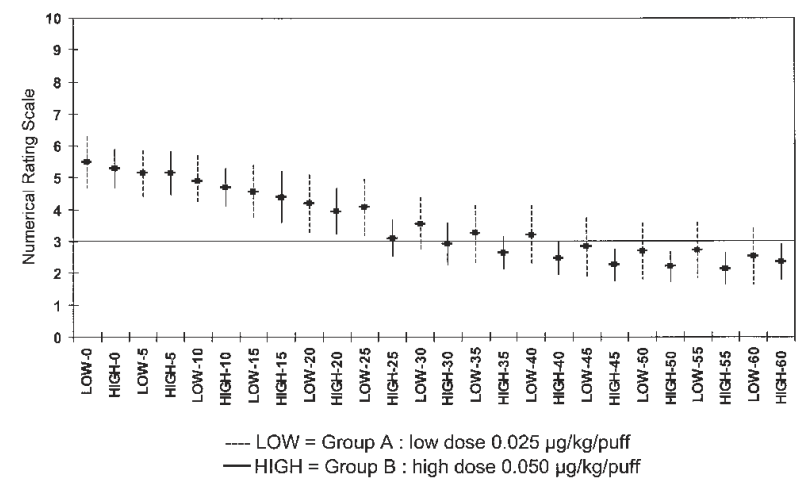

FIGURE 3 Numerical rating scale scores for pain, showing the mean value and 95\% confidence interval (CI95), allowing determination of whether the CI95 for a group at any given time lies entirely within the zone defined as analgesic success $(0-3)$. The abscissa line represents five-minute time intervals for Groups A and B.

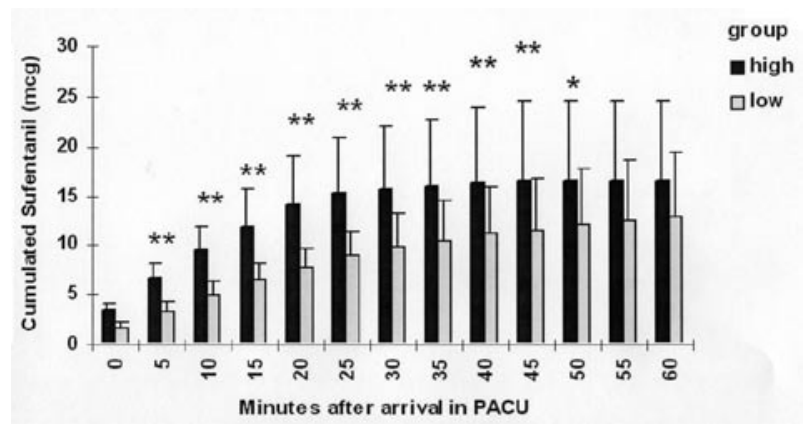

FIGURE 4 The cumulative amount of sufentanil delivered as a function of time for the two groups: ${ }^{*} P<0.01$ high dose $v$ low dose, ${ }^{*} P<0.05$ high dose $v s$ low dose.

scale and the nausea scale were recorded every five minutes. Side effects, including nausea, vomiting, pruritus or dysphoria were also documented. The protocol ended when the patient left the PACU.

Data were compared using the Fisher's exact test, the Mann-Whitney U test or the Student's t test as appropriate. Heart rate, respiratory rate, systolic and diastolic blood pressures were compared using a twoway analysis of variance for repeated measures. The evolution of the NRS score for pain in the two groups was analyzed using the Log-Rank test, and graphically depicted using the Kaplan-Meier method.

The NRS score for pain is also presented graphically, showing the mean value and $95 \%$ confidence interval (CI95), allowing to evaluate whether the 

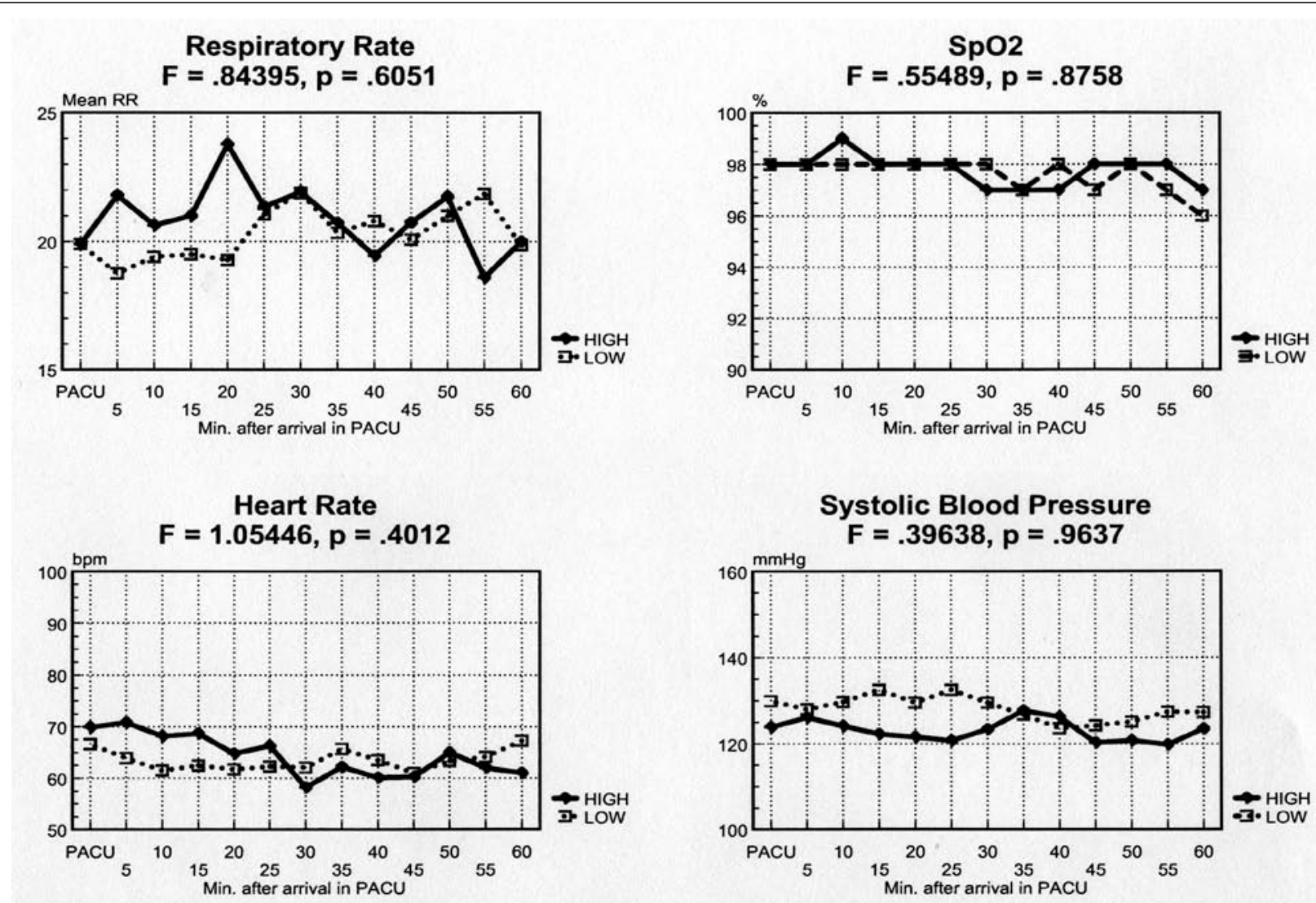

FIGURE 5 Mean values of respiratory rate, oxygen saturation, systolic blood pressure and heart rate are shown. Values remained stable throughout the observation period, with no intergroup differences.

CI95 for a group at a given time lies entirely within the zone defined as analgesic success (NRS pain score $0-3) .{ }^{12}$ All values are presented as mean \pm standard deviation, or as number of patients. For all tests, $P<$ 0.05 was considered statistically significant.

\section{Results}

Forty-five patients were included in the study. Forty patients were randomized and five patients were not included because they did not reach a pain NRS score $>3$.

Demographic data are presented in Table II. No statistical difference was observed between both groups with respect to gender, age, weight, type of surgery (hemorrhoidectomy or herniorrhaphy), duration of surgery and the selected opioid (fentanyl or sufentanil) used during surgery. The total dose of sufentanil or fentanyl given during surgery and the NRS values upon arrival in the PACU were similar in both groups.
Figure 2 illustrates the probability of persistence of NRS $>3$. This was consistently lower in Group B compared to Group A $(P=0.015)$, with the greatest difference observed after $20 \mathrm{~min}$. At $60 \mathrm{~min}$, no patient had a NRS pain score $>3$ in Group B, whereas there was still a probability of $20 \%$ of patients having a NRS > 3 in Group A.

Figure 3 shows the CI95 intervals during the stay in the PACU. The CI95 lies entirely within the zone defined as analgesic success (NRS between $0-3$ ) at 40 min. This situation was never obtained after $60 \mathrm{~min}$ in Group A. The number of boluses or puffs given during the first hour was $7.2 \pm 3.8$ and $4.7 \pm 2.2$ in Groups A and $\mathrm{B}$, respectively $(P=0.017)$.

Figure 4 shows the cumulated quantity of sufentanil expressed as a function of time in both groups. The dose of sufentanil was significantly less in Group A than in Group B before $\mathrm{t}=55 \mathrm{~min}$. 
Two patients in Group A required rescue therapy, but none in Group B. One patient required three boluses of $2 \mathrm{mg}$ morphine iv, and the other patient required seven boluses of $2 \mathrm{mg}$ of morphine $i v$ as rescue therapy. Oxygen saturation, respiratory rate, blood pressure and heart rate remained stable throughout the observation period, with no intergroup differences (Figure 5). No patient vomited, and the NRS for nausea was similar in both groups. During their stay in the PACU, all patients were evaluated as having a value of 2 on the Ramsay sedation scale.

Finally, given the probability of persistence of NRS $>3$ of $0 \%$ in Group B and 20\% in Group A, a sample size of 20 from each group gives a probability of 0.339 of rejecting the null hypothesis of equal proportions if the alternative holds.

\section{Discussion}

The results of this study show that a dose of 0.05 $\mu \mathrm{g} \cdot \mathrm{kg}^{-1}$ of IN sufentanil, with a minimum time of five minutes between doses, was more rapidly effective and required fewer doses to achieve adequate postoperative pain management compared to a dose of $0.025 \mu \mathrm{g} \cdot \mathrm{kg}^{-1}$ sufentanil IN. After $60 \mathrm{~min}$, the cumulated doses were similar in both groups, but an NRS pain score $<4$ was more rapidly attained with a dose of $0.050 \mu \mathrm{g} \cdot \mathrm{kg}^{-1}$. Moreover, the safety profile of the higher dose is similar to that of the lower dose in the PACU, as reflected by the stability of the hemodynamic, respiratory parameters and Ramsay sedation scores throughout the observation period, in both groups. Vomiting episodes and NRS for nausea were also similar in both groups.

Our results correlate with those of the study of Helmers et al. published in $1989 .^{7}$ In their study, the absorption and sedation following an IN dose of 15 $\mu \mathrm{g}$ of sufentanil were evaluated and compared with the same dose given intravenously. Following IN administration, sufentanil blood concentrations after five and ten minutes were 36 and $56 \%$ of those attained after iv injection, respectively. After 30,60 and $120 \mathrm{~min}$, there were no significant differences between the sufentanil blood concentrations, and sedation associated with either dosing route.

In our study, four puffs of $0.05 \mu \mathrm{g} \cdot \mathrm{kg}^{-1}$ of sufentanil IN could be delivered within an interval of 20 min, which corresponds to a cumulative dose of 0.2 $\mu \mathrm{g} \cdot \mathrm{kg}^{-1}$. For a patient weighing $70 \mathrm{~kg}$, this IN dose of $14 \mu \mathrm{g}$ of sufentanil could be comparable to the $15 \mu \mathrm{g}$ IN sufentanil puff dose given in the study of Helmers. ${ }^{7}$ However, this remains hypothetical, as the pharmacokinetics of our delivery system and drug bioavailability were not studied.
Another important aspect of our study is the use of a potent opioid with a high concentration (sufentanil $50 \mu \mathrm{g} \cdot \mathrm{mL}^{-1}$ ). Indeed, the maximum volume for an adult to avoid run-off into the pharynx by a single puff administration in one nostril is considered as being $150 \mu \mathrm{L} .{ }^{1}$ For this reason, available puff devices allow delivery of constant low volumes such as the $109 \pm 3 \mu \mathrm{L}$ measured in the puff device used in this study. Using a fixed volume spray delivery device, the concentration of IN sufentanil administered was different, and easily adapted for the weight of the patient with a simple formula.

The delivering device used in this study does not allow for a change of delivered volume. Moreover, it is not possible to lock this device to limit the number of doses per unit time. The limitation of this form of administration is the inter-individual variation in pharmacokinetics of intranasally administered opioids. In addition, long-term use can be associated with nasal congestion, epistaxis, nasal irritation or infection. ${ }^{1}$

In conclusion, repeated administration of IN sufentanil $0.05 \mu \mathrm{g} . \mathrm{kg}^{-1}$ to adult patients was more effective than $0.025 \mu \mathrm{g} \cdot \mathrm{kg}^{-1}$. All patients given the higher dose had satisfactory analgesia within one hour after admission to the PACU, without adverse respiratory or hemodynamic effects.

\section{References}

1 Dale $O$, Hjortkjaer R, Kharasch ED. Nasal administration of opioids for pain management in adults. Acta Anaesthesiol Scand 2002; 46: 759-70.

2 Gillis JC, Benfield P, Goa KL. Transnasal butorphanol. A review of its pharmacodynamic and pharmacokinetic properties, and therapeutic potential in acute pain management. Drugs 1995; 50: 157-75.

3 Striebel HW, Koenigs D, Kramer J. Postoperative pain management by intranasal demand-adapted fentanyl titration. Anesthesiology 1992; 77: 281-5.

4 Striebel HW, Pommerening J, Rieger A. Intranasal fentanyl titration for postoperative pain management in an unselected population. Anaesthesia 1993; 48: 753-7.

5 Striebel HW, Oelmann T, Spies C, Rieger A, Schwagmeier $R$. Patient-controlled intranasal analgesia: a method for noninvasive postoperative pain management. Anesth Analg 1996; 83: 548-51.

6 Toussaint S, Maidl J, Schagmeier R, Striebel HW. Patient-controlled intranasal analgesia: effective alternative to intravenous PCA for postoperative pain relief. Can J Anesth 2000; 47: 299-302.

7 Helmers JH, Noorduin H, Van Peer A, Van Leeuwen L, Zuurmond $W W$. Comparison of intravenous and intranasal sufentanil absorption and sedation. Can J Anaesth 1989; 36: 494-7. 
8 Henderson JM, Brodsky DA, Fisher DM, Brett CM, Hertzka RE. Pre-induction of anesthesia in pediatric patients with nasally administered sufentanil. Anesthesiology 1988; 68: 671-5.

9 Vercauteren M, Boeckx E, Hannegreefs G, Noorduin H, Vanden Bussche $G$. Intranasal sufentanil for pre-operative sedation. Anaesthesia 1988; 43: 270-3.

10 Zedie N, Amory DW, Wagner BK, O'Hara DA. Comparison of intranasal midazolam and sufentanil premedication in pediatric outpatients. Clin Pharmacol Ther 1996; 59: 341-8.

11 Karl HW, Keifer AT, Rosenberger JL, Larach $M G$, Ruffle JM. Comparison of the safety and efficacy of intranasal midazolam or sufentanil for preinduction of anesthesia in pediatric patients. Anesthesiology 1992; 76: 209-15.

12 Mantha S, Thisted R, Foss J, Ellis JE, Roizen M. A proposal to use confidence intervals for visual analog scale data for pain measurement to determine clinical significance. Anesth Analg 1993; 77: 1041-7. 